\title{
A POLAR REPRESENTATION OF SINGULAR MATRICES
}

\author{
BY JOHN WILLIAMSON
}

Let $A=\left(a_{i j}\right),(i=1,2, \cdots, m ; j=1,2, \cdots, n)$, be a matrix of $m$ rows and $n$ columns, whose elements $a_{i j}$ are complex numbers. It has been shown $\dagger$ that, if $m=n$ and $A$ is non-singular, $A=P_{1} U=U P_{2}$, where $U$ is a unitary matrix, while $P_{1}$ and $P_{2}$ are positive definite hermitian matrices. Moreover in such a polar representation of $A$, as it has been called, the matrices $P_{1}, P_{2}$, and $U$ are uniquely determined. We shall show that, if $m=n$ and the rank of $A$ is $r<n, A=P_{1} U=U P_{2}$, where $P_{1}$ and $P_{2}$ are uniquely determined positive hermitian matrices of rank $r$ and $U$ is unitary but no longer unique. Any such representation of course is impossible if $m \neq n$, as by definition both hermitian and unitary matrices are square, but it will be shown that somewhat analogous results exist in this case as well.

As is customary we shall denote the conjugate transposed of $A$ by $A^{*}=\left(a_{i j}^{*}\right)$, where $a_{j}^{*}=\bar{a}_{j i}$, the complex conjugate of $a_{j i}$. We shall use this notation, even if $A$ is a vector, that is, a matrix of one row, so that in this case $A A^{*}$ will simply denote the norm of the vector $A$. For the sake of brevity we shall use the notations $E_{j}$ for the unit matrix of order $j$ and $0_{i, j}$ for the zero matrix of $i$ rows and $j$ columns.

The matrix $N_{1}=A A^{*}$ is a square matrix of order $m$ and the matrix $N_{2}=A^{*} A$ is a square matrix of order $n$, and since $N_{1}=N_{1}^{*}$ and $N_{2}=N_{2}^{*}$, both of these matrices are hermitian. Moreover, if the rank of $A$ is $r$, the rank of $N_{1}$ is $r$ and so is the rank of $N_{2}$. For, if $K$ is the $r$ th compound $\ddagger$ of $A$, at least one element $k_{i j}$ of $K$ is different from zero. The element in the $i$ th place of the leading diagonal of the product matrix $K K^{*}$ is $\sum_{t} k_{i t} \bar{k}_{i t}$, which is a positive real number, since $k_{i j}$ is not zero. Accordingly there is at least one $r$-rowed determinant of $N_{1}$

$\dagger$ L. Autonne, Bulletin de la Société Mathématique, vol. 30 (1902), pp. 121-134. A. Wintner and F. D. Murnaghan, On a polar representation of nonsingular matrices, Proceedings of the National Academy of Sciences, vol. 17 (1931), pp. 676-678.

$\ddagger$ Turnbull and Aitken, The Theory of Canonical Matrices, p. 27. 
which is not zero, so that the rank of $N_{1}$ is at least $r$. Since the rank of $N_{1}$ cannot exceed the rank $r$ of $A$, the rank of $N_{1}$ is exactly $r$. Similarly the rank of $N_{2}$ is $r$.

Since $N_{1}$ is a hermitian matrix of rank $r$, there exists an $m$ rowed unitary matrix $X$ such that $\dagger$

$$
X N_{1} X^{*}=D=\left(\begin{array}{ll}
D_{11} & 0_{r, m-r} \\
0_{m-r, r} & 0_{m-r, m-r}
\end{array}\right),
$$

where $D_{11}$ is a diagonal matrix of order $r$. If $B=X A, B^{*}=A^{*} X^{*}$, so that

$$
B B^{*}=X A A^{*} X^{*}=X N_{1} X^{*}=D .
$$

If we denote the row vectors of $B$ by $b_{i},(i=1,2, \cdots, m)$, the column vectors of $B^{*}$ are $b_{i}^{*},(i=1,2, \cdots, m)$, and the element in the $i$ th row and $j$ th column of $B B^{*}$ is $b_{i} b_{j}{ }^{*}$. It therefore follows from (1) and (2) that $b_{i} b_{i}^{*}=0,(i=r+1, \cdots, m)$. Hence $B$ is a matrix whose last $m-r$ rows are zero so that

$$
B=\left(\begin{array}{l}
B_{1} \\
0_{m-r, n}
\end{array}\right)
$$

where $B_{1}$ is a matrix of $r$ rows and $n$ columns. By a similar argument applied to $N_{2}$ instead of to $N_{1}$, it can be shown that there exists an $n$-rowed unitary matrix $Y$ such that

$$
A Y=\left(\begin{array}{ll}
B_{2} & 0_{m, n-r}
\end{array}\right),
$$

where $B_{2}$ is a matrix of $m$ rows and $r$ columns. From (3) and (4) we deduce that

$$
X A Y=C=\left(\begin{array}{ll}
C_{11} & 0_{r, n-r} \\
0_{m-r, r} & 0_{m-r, n-r}
\end{array}\right),
$$

where $C_{11}$ is an $r$-rowed square matrix, which is non-singular since the rank of $A$ is $r$. Since $X N_{1} X^{*}=X A Y Y^{*} A^{*} X^{*}=C C^{*}$, it follows from (1) and (5) that

$$
C_{11} C_{11} *=D_{11} \text {. }
$$

Denoting by $c_{i},(i=1,2, \cdots, r)$, the row vectors of $C_{11}$, and by $d_{i}$ the element in the $i$ th place of the diagonal matrix $D_{11}$, we

$\dagger$ Turnbull and Aitken, op. cit., p. 85. 
deduce the equalities $c_{i} c_{j}^{*}=0, \quad(i \neq j), \quad c_{i} c_{i}^{*}=d_{i}, \quad(i, j=1$, $2, \cdots, r)$. Hence $d_{i}$ is a positive real number and the vector $c_{i} / d_{i}{ }^{1 / 2}$ is a normalized vector. Consequently the matrix

$$
V_{11}=\left(\begin{array}{cccc}
d_{1}^{-1 / 2} & 0 & \cdots & 0 \\
0 & d_{2}^{-1 / 2} & \cdots & 0 \\
\cdot & \cdot & \cdots & \cdot \\
\cdot & \cdot & \cdots & \cdot \\
0 & 0 & \cdots & d_{r}^{-1 / 2}
\end{array}\right) C_{11}=\left(D_{11}\right)^{-1 / 2} C_{11}
$$

is unitary and

$$
C_{11}=Q_{11} V_{11}
$$

where $V_{11}$ is a unitary matrix of order $r$ and $Q_{11}=\left(D_{11}\right)^{1 / 2}$ is a positive definite hermitian matrix of order $r$. Using the value of $C$ given by (5), we have, from (7),

$$
\begin{aligned}
C & =\left(\begin{array}{ll}
Q_{11} V_{11} & 0_{r, n-r} \\
0_{m-r, r} & 0_{m-r, n-r}
\end{array}\right) \\
& =\left(\begin{array}{ll}
Q_{11} & 0_{r, n-r} \\
0_{m-r, r} & 0_{m-r, n-r}
\end{array}\right)\left(\begin{array}{ll}
V_{11} & 0_{r, n-r} \\
0_{n-r, r} & E_{n-r}
\end{array}\right) .
\end{aligned}
$$

Now if $m \leqq n$, this last result may be written in the form

$$
C=\left(Q \quad 0_{m, n-m}\right) V,
$$

where

$$
Q=\left(\begin{array}{ll}
Q_{11} & 0_{r, m-r} \\
0_{m-r} & 0_{m-r, m-r}
\end{array}\right), \quad V=\left(\begin{array}{ll}
V_{11} & 0_{r, n-r} \\
0_{n-r, r} & E_{n-r}
\end{array}\right),
$$

$Q$ being a positive hermitian matrix of order $m$ and rank $r$, while $V$ is a unitary matrix of order $n$. Moreover, if

$$
C=\left(Q_{1} \quad 0_{m, n-m}\right) V_{1}
$$

is another such representation of $C$, where $Q_{1}$ is a positive hermitian matrix of order $m$ and rank $r$ and $V_{1}$ is a unitary matrix of order $n$, we see that $C C^{*}=Q_{1} Q_{1}^{*}=Q_{1}^{2}=D$, so that $Q_{1}=D^{1 / 2}=Q$. Accordingly we may write (10) in the form

$$
C=\left(\begin{array}{ll}
Q_{11} & 0_{r, n-r} \\
0_{m-r, r} & 0_{m-r, n-r}
\end{array}\right)\left(\begin{array}{ll}
W_{11} & V_{12} \\
V_{21} & V_{22}
\end{array}\right),
$$


where $W_{11}$ is an $r$-rowed square matrix, $V_{22}$ an $(n-r)$-rowed square matrix, $V_{12}$ an $r$ by $n-r$ and $V_{21}$ an $n-r$ by $n$ matrix. Hence

$$
C=\left(\begin{array}{ll}
Q_{11} W_{11} & Q_{11} V_{12} \\
0_{m-r, r} & 0_{m-r, n-r}
\end{array}\right),
$$

and comparison with (8) shows that $W_{11}=V_{11}$ and $V_{12}=0_{r, n-r}$. Since $V_{1} V_{1}^{*}=E_{n}$, it follows easily that $V_{21}=0_{n-r, r}$ and that $V_{22} V_{22}{ }^{*}=E_{n-r}$. Hence the matrix $V_{1}$ in (10) is of the form $V W$, where

$$
W=\left(\begin{array}{ll}
E_{r} & 0_{r, n-r} \\
0_{n-r, r} & V_{22}
\end{array}\right)
$$

and $V_{22}$ is an arbitrary unitary matrix of order $n-r$. We have therefore proved the following lemma.

LEMmA. The matrix $C$ can be represented in the form $C=\left(\begin{array}{ll}Q & 0_{n-m}\end{array}\right) V_{1}$, where $Q$ is a positive hermitian matrix of order $m$ and rank $r$ and $V_{1}$ is a unitary matrix of order $n$. The matrix $Q$ is unique while the matrix $V_{1}$ is one of a set $\left[V_{1}\right]=[V W]$, where $V$ is a fixed unitary matrix and $W$ ranges over a group $G$ of unitary matrices of order $n$ simply isomorphic with the group of all unitary matrices of order $n-r$.

Since $A=X^{*} C Y^{*}$, by (9),

$$
A=X^{*}\left(Q \quad 0_{m, n-m}\right) V Y^{*}=X^{*}\left(Q \quad 0_{m, n-m}\right) X_{1} X_{1}^{*} V Y^{*},
$$

where

$$
X_{1}=\left(\begin{array}{ll}
X & 0_{m, n-m} \\
0_{n-m, m} & E_{n-m}
\end{array}\right)
$$

Hence

$$
A=\left(\begin{array}{ll}
X^{*} Q X & 0_{m, n-m}
\end{array}\right) X_{1}^{*} V Y^{*}=\left(\begin{array}{ll}
P & 0_{m, n-m}
\end{array}\right) U,
$$

where $P=X^{*} Q X$ is a positive hermitian matrix of order $m$ and rank $r$, while $U=X_{1}^{*} V Y^{*}$ is a unitary matrix of order $n$. If $A=\left(\begin{array}{ll}P_{1} & 0_{m, n-m}\end{array}\right) U_{1}$ is another such representation of $A$, it follows easily from the previous lemma that $X P_{1} X^{*}=Q$, so that $P_{1}=P$, and that $U_{1}=U Z$, where $Z=Y W Y^{*}$. Accordingly we have proved the following theorem. 
THEOREM. If $A$ is a matrix of $m$ rows and $n$ columns of rank $r$ and $m \leqq n, A$ can be represented in the form

$$
A=\left(\begin{array}{ll}
P_{1} & 0_{m, n-m}
\end{array}\right) U_{1},
$$

where $P_{1}$ is a positive hermitian matrix of order $m$ and rank $r$ and $U_{1}$ is a unitary matrix of order $n$. The matrix $P_{1}$ is unique while the matrix $U_{1}$ is one of a set $\left[U_{1}\right]=\left[U Z_{1}\right]$, where $U$ is a fixed unitary matrix and $Z_{1}$ one of a group $G_{1}$ of matrices, simply isomorphic with the group of all unitary matrices of order $n-r$.

COROLlaRy. Under the above hypotheses the totality of unitary matrices $Z_{1}$ for which $A Z_{1}=A$ forms a group simply isomorphic with the group of all unitary matrices of order $n-r$. This group is the group $G_{1}$.

For if $A Z_{1}=A,\left(\begin{array}{ll}P_{1} & 0_{m, n-m}\end{array}\right) U Z_{1}=\left(\begin{array}{ll}P_{1} & 0_{m, n-m}\end{array}\right) U$, and $Z^{1}$ must lie in $G_{1}$. Similarly if $Z_{1}$ lies in $G_{1}, A Z_{1}=A$.

If $m \geqq n, A^{*}$ is a matrix in which the number of its rows is at most equal to the number of its columns. Accordingly, under this hypothesis our theorem is true if $A$ is replaced by $A^{*}$. Hence, if $m \geqq n, A$ can be represented in the form

$$
A=U_{2}\left(\begin{array}{l}
P_{2} \\
0_{m-n, n}
\end{array}\right),
$$

where $P_{2}$ is a uniquely determined positive hermitian matrix of order $n$ and rank $r$ and $U_{2}$ is one of a set $\left[U_{2}\right]=\left[Z_{2} U\right]$, where $Z_{2}$ ranges over a group $G_{2}$ simply isomorphic with the group of all unitary matrices of order $m-r$.

When $m=n$, that is, when the matrix $A$ is square, some further results follow. In this case equations (11) and (12) become

$$
A=P_{1} U_{1},
$$

and

$$
A=U_{2} P_{2},
$$

respectively, where $P_{1}$ and $P_{2}$ are positive hermitian matrices of rank $r$ and $U_{1}$ and $U_{2}$ are unitary matrices. The two groups $G_{1}$ and $G_{2}$ are simply isomorphic; the two sets $\left[U_{1}\right]$ and $\left[U_{2}\right]$ coincide; if $U_{1}$ belongs to the set $\left[U_{1}\right], P_{2}=U_{1}^{*} P_{1} U_{1}$. The first 
statement is obviously true; if $U_{1}$ lies in $\left[U_{1}\right], A=U_{1} U_{1}^{*} P_{1} U_{1}$ and, since $U_{1}^{*} P_{1} U_{1}$ is a positive hermitian matrix of rank $r$, $U_{1}^{*} P_{1} U_{1}=P_{2}$ and $U_{1}$ lies in $\left[U_{2}\right]$. Similarly any member $U_{2}$ of $\left[U_{2}\right]$ lies in $\left[U_{1}\right]$. Further the matrix $P_{2}$ is invariant under unitary transformation by any matrix of the group $G_{1}$, and $P_{1}$ under transformation by any matrix of the group $G_{2}$. For if $Z_{1}$ lies in $G_{1}, A Z_{1}=A$ so that $A=U_{2} Z_{1} Z_{1}{ }^{*} P_{2} Z_{1}$, and accordingly, $Z_{1}^{*} P_{2} Z_{1}=P_{2}$.

The Johns Hopkins University

\section{ON A THEOREM OF FERAUD}

BY D. C. LEWIS, JR.*

The Birkhoff-Pfaffian equations of dynamics are written in variational form as follows:

$$
\delta \int\left[\sum_{i=1}^{2 m} X_{i}\left(\frac{d x_{i}}{d t}\right)+Q\right] d t=0,
$$

where $Q$ and the $X$ 's are functions of $x_{1}, \cdots, x_{2 m}$ and, in general, depend also periodically upon $t$, and where the skew-symmetric determinant $\left|a_{i j}\right|,\left(a_{i j}=\partial X_{i} / \partial x_{j}-\partial X_{j} / \partial x_{i}\right)$, does not vanish in the regions considered. We restrict attention to the neighborhood of a generalized equilibrium point, that is, a point where all the $\partial Q / \partial x_{i}-\partial X_{i} / \partial t$ vanish identically in $t$. We take this point at the origin, $x_{i}=0,(i=1,2, \cdots, 2 m)$.

The problem of reducing the Pfaffian system to a Hamiltonian system can be reduced to that of finding a non-singular transformation, $x_{i}=x_{i}\left(y_{1}, \cdots, y_{2}\right)$, leaving the origin invariant (and depending in general periodically upon $t$ ) which reduces the linear differential form $\sum_{i=1}^{2 m} X_{i} d x_{i}$ to the form $\sum_{i=1}^{m} y_{2 i} d y_{2 i-1}$ $+d w$, where $d w$ is an exact differential in $y_{1}, \cdots, y_{2}$, the coefficients of which are independent of $t$. This same problem also will play an important role in a future paper of mine on "conservative" transformations in $2 m$-dimensional spaces.

The problem has been considered by Féraud, $\uparrow$ who obtained a

* National Research Fellow.

$\dagger$ Extension au cas d'un nombre quelconque de degrés de liberté d'une propriété relative aux systèmes Pfaffiens, Comptes Rendus, vol. 190 (1930), pp. 358-360. 